\title{
ChemComm
}

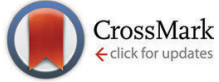

Cite this: Chem. Commun., 2015, 51,5467

Received 20th October 2014,

Accepted 4th November 2014

DOI: $10.1039 / c 4 c c 08285 \mathrm{k}$

www.rsc.org/chemcomm

\section{A bio-catalytically driven Janus mesoporous silica cluster motor with magnetic guidance $\dagger$}

\author{
Xing Ma and Samuel Sanchez*
}

\begin{abstract}
A bio-catalytic Janus motor based on the mesoporous silica cluster (JMSC) is fabricated. Chemically conjugated catalase triggers the decomposition of $\mathrm{H}_{2} \mathrm{O}_{2}$ to produce driving force by bubble propulsion, while a metallic (Ni) coating layer allows for magnetic guidance of the motor. The JMSC motor can act as a delivery vehicle with cargo loading inside its mesopores.
\end{abstract}

Janus structures have been employed to fabricate micro/nanomotors (MNMs), ${ }^{1,2}$ since distinguished surface properties of the two "faces" can produce a "net force" for the movement of Janus motors, such as catalytic reaction induced electrolyte/ ionic self-diffusiophoresis, ${ }^{3-8}$ bubble propulsion, ${ }^{9-12}$ or neutral self-diffusiophoresis. ${ }^{13-15}$ At present, most Janus motors are mainly fabricated with solid spheres, like silica $\left(\mathrm{SiO}_{2}\right)$ and polystyrene (PS) particles. ${ }^{1,16}$ The rigid properties of such motors limit their delivery capability, since cargos can only be loaded on Janus particles' outer surface. Furthermore, requirements of catalytic sites on motors' surface further minimize cargo loading capability. Thus, the use of rigid Janus motors is limited as effective delivery vehicles. Mesoporous silica nanoparticles (MSNPs) have been widely investigated as catalyst supports ${ }^{17,18}$ and drug/gene delivery carriers, ${ }^{19,20}$ in virtue of their unique structure, including tunable size, high surface area, uniform pore size and high pore volume, ${ }^{21,22}$ which allows for cargo molecule loading in large quantity. ${ }^{23} \mathrm{So}$, it is of great interest to fabricate Janus motors with mesoporous silica materials, ${ }^{24}$ considering their excellent capability of cargo loading and delivery.

As an important mechanism, bubble propulsion has been utilized to provide driving forces for micro/nano-motors (MNMs). ${ }^{11,25-27}$ Continuous production of bubbles is essential for the motors' movement by the bubble propulsion mechanism. For example, in the case of micro-tube based jets, the internal

Max Planck Institute for Intelligent Systems, Heisenbergstraße 3, 70569 Stuttgart, Germany.E-mail: sanchez@is.mpg.de

$\dagger$ Electronic supplementary information (ESI) available. See DOI: 10.1039/ c4cc08285k catalytic metal platinum (Pt) layer can decompose $\mathrm{H}_{2} \mathrm{O}_{2}$ and bubbles are formed inside the micro-sized channels to provide the driving force for the jet movement. ${ }^{27-29}$ However, for Janus particles based motors, bubbles can hardly form on the smooth surface of spherical particles, and usually a rough surface with small cavities is preferred for bubble generation. ${ }^{12,30}$ During the classic "sol-gel" synthesis of MSNPs by using $\mathrm{NaOH}$ as a catalyst, it is a great challenge to obtain completely monodispersed MSNP. Thus, considerable amounts of mesoporous silica cluster (MSC) are present due to "unwanted" aggregations. ${ }^{31,32}$ However, the other side of the coin is that small cavities or narrow gaps between aggregated MSNPs in these MSCs might be able to serve as a rough surface and provide ideal nucleation sites for the bubble generation and local accumulation, thus driving the movement of motors by bubble propulsion.

Therefore, taking advantage of such an "unwanted" aggregation "problem" during the classic synthesis of MSNPs, we propose a novel Janus mesoporous silica cluster (JMSC) based motor which is driven by bio-catalytic reaction induced bubble propulsion. Hereby, a micro-sized MSC consisting of MSNPS with size less than $100 \mathrm{~nm}$ was fabricated (Fig. 1). Via surface

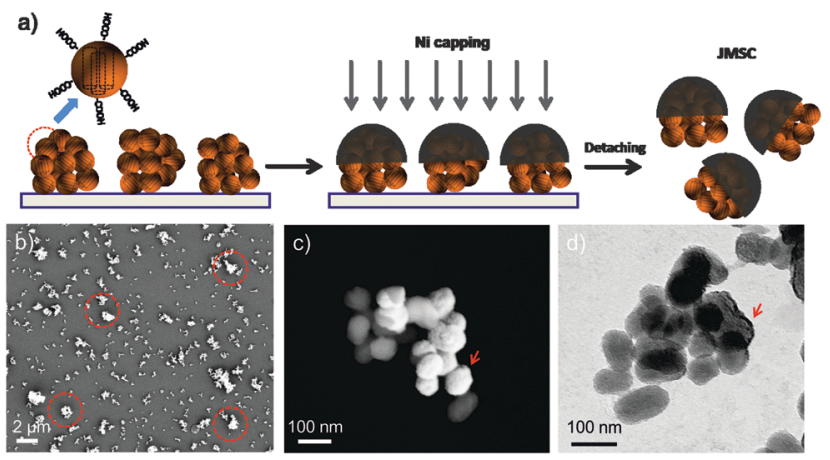

Fig. 1 (a) Schematic illustration of Janus mesoporous silica cluster (JMSC) fabrication; (b) SEM images of a MSC monolayer; (c) SEM and (d) TEM images of a JMSC with $\mathrm{Ni}(10 \mathrm{~nm})$ coating by electron beam deposition. 
chemistry functionalization, the MSC was initially modified with carboxylic acid groups. Then, one side of the MSC was covered with a magnetic metal layer (Ni) by electron-beam (E-beam) deposition, yielding a JMSC. The magnetic layer (Ni) can be used for external magnetic guidance of the JMSC motors' movement. ${ }^{14}$ Catalase enzyme was conjugated to the uncovered side of the JMSC to catalyse the decomposition reaction of $\mathrm{H}_{2} \mathrm{O}_{2} \cdot{ }^{33}$ The rough surface with small cavities and gaps at the uncovered side of the JMSC motors can provide possible nucleation sites for $\mathrm{O}_{2}$ bubble generation. More importantly, unlike other Janus motors by using solid spheres, the mesoporous structure of the JMSC motors have uniform nano-channels with high surface area and large pore volume, which can be used for cargo molecules loading and thus realize cargo delivery by the bio-catalytic reaction driven JMSC motors.

MSCs were fabricated by a modified sol-gel process that was used to prepare mesoporous silica nanoparticles..$^{23,34,35}$ Typically, silica precursor TEOS was added into an aqueous base solution containing catalyst $\mathrm{NaOH}$ and surfactant cetyltrimethylammonium bromide (CTAB) under vigorous stirring. After $1 \mathrm{~h}$ reaction, a small amount of additional TEOS was added to induce more aggregation of MSNPs under mild stirring, yielding more MSCs than under normal conditions. Then, the MSC was suspended in EtOH solution containing (3-aminopropyl)triethoxysilane (APTES) and stirred for $24 \mathrm{~h}$ to produce the amino modified MSC, noted as MSC-NH $\mathrm{N}_{2}$. From the FT-IR spectra of $\mathrm{MSC}^{-\mathrm{NH}_{2}}$ (red curve in Fig. 2a), a new peak at $1520 \mathrm{~cm}^{-1}$ due to the appearance of a primary amine. ${ }^{36}$ And the zeta potential value was reversed from a negative value $(-37.6 \pm 6.10 \mathrm{mV})$ of bare MSC to a positive value $(39.5 \pm 5.30 \mathrm{mV})$ of $\mathrm{MSC}-\mathrm{NH}_{2}$. Then, the $\mathrm{MSC}-\mathrm{NH}_{2}$ was converted into carboxylic acid groups $(-\mathrm{COOH})$ functionalized MSC, noted as MSC-COOH. Extra peaks at $1726 \mathrm{~cm}^{-1}$ contributed by $-\mathrm{COOH}$ groups were found in the FT-IR spectra of MSC-COOH. Another new peak at $1560 \mathrm{~cm}^{-1}$ due to the amide bond proved the conjugation process between the primary amine and succinic anhydride. ${ }^{36-38}$ The zeta potential value of MSC-COOH was again reversed to a negative value $(-44.5 \pm$ $6.49 \mathrm{mV}$ ), indicating successful functionalization of carboxylic acid groups on the surface of MSC-COOH.

Before E-beam deposition, a monolayer of MSC-COOH was first prepared (Fig. 1a) by dipping in ethanol solution containing MSC-COOH a clean glass slide that had been pre-treated with oxygen plasma to make it hydrophilic and ensure the good spreading of the dipped MSC-COOH solution. The prepared
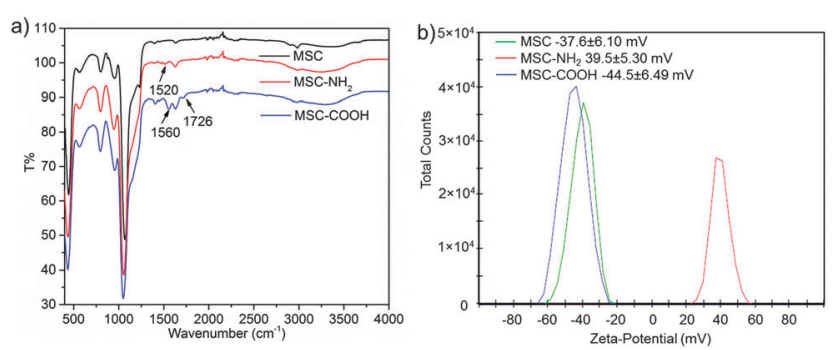

Fig. 2 (a) FT-IR spectra and (b) zeta-potential values of a MSC, MSC-NH and $\mathrm{MSC}-\mathrm{COOH}$ indicate the surface functionalization process. monolayer of MSC-COOH was observed by scanning electron microscopy (SEM) (Fig. 1b). The presence of micro-sized MSCs with irregular shapes was indicated by red circles. The magnetic layer of Ni of about $15 \mathrm{~nm}$ was deposited and the obtained JMSC with Ni coating, denoted as JMSC@Ni, was collected by sonication in DI $\mathrm{H}_{2} \mathrm{O}$. After E-beam deposition, the Ni coating layer was clearly observed by both SEM and transmission electron microscopy (TEM) images, as shown in Fig. 1b and c. From the SEM and TEM images, the size of MSNPs that comprise the MSCs was found to be around $80 \mathrm{~nm}$. More importantly, the aggregation of these MSNPs produced a rough surface of MSCs with small cavities and gaps between MSNPs, which is critical for bubble generation.

Afterwards, catalase enzyme was conjugated to the non-coated side of the JMSC@Ni via 1-ethyl-3-(3-dimethylaminopropyl)carbodiimide (EDC)- $N$-hydroxysuccinimide (NHS) chemistry. The carboxylic acid groups were first activated by stirring the JMSC@Ni in PBS buffer ( $\mathrm{pH}=6.0$ ) containing EDC-NHS for $1 \mathrm{~h}$. Then, catalase aqueous solution was added and the mixture solution was adjusted to slightly basic conditions with a $\mathrm{pH}$ of about 8.5 by adding an appropriate amount of dilute $\mathrm{NaOH}$ aqueous solution. After $24 \mathrm{~h}$ reaction, the catalase conjugated JMSC@Ni, denoted as JMSC@Ni-catalase, was collected by centrifugation and washed with deionized (DI) $\mathrm{H}_{2} \mathrm{O}$ (Fig. 3a).

The cargo loading capability of the JMSC motors was tested. An FITC labelled JMSC was first fabricated. The fluorescent dye Rhodamine B (Rh.B) was chosen as a model cargo. The JMSC(FITC)@Ni-catalase was suspended in Rh.B solution $\left(0.5 \mathrm{mg} \mathrm{mL}^{-1}\right)$. After stirring for $24 \mathrm{~h}$ to ensure the cargo molecule loading into the mesopores by free diffusion, the Rh.B loaded JMSC(FITC)@catalase was collected by centrifugation and washed with DI $\mathrm{H}_{2} \mathrm{O}$ several times. Then, confocal laser scanning microscopy (CLSM) was used to confirm the loading of the cargo molecule inside the JMSC motors. From Fig. 3b, we found that red fluorescence dots due to the presence of Rh.B cargo molecules were completely overlapped with the green fluorescence dots representing locations of the FITC labelled JMSC motors, as we only find yellow dots (indicated by white arrows) in the overlay image, which certified the cargo loading inside the JMSC motor.

a)

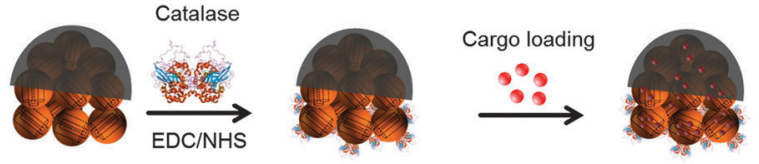

b)

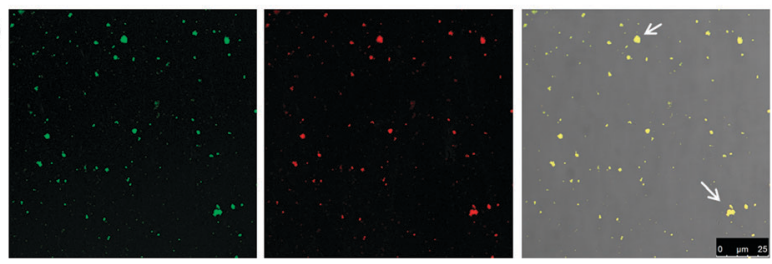

Fig. 3 (a) Schematic illustration of catalase conjugation on the non-coated side of a JMSC and further cargo (Rh.B) loading inside the mesopores of a JMSC motor. (b) CLSM images of a Rh.B loaded JMSC motor. From left to right are the green channel (FITC), the red channel (Rh.B), and an overlay of the bright field, green and red channels (scale bar $=25 \mu \mathrm{m}$ ). 


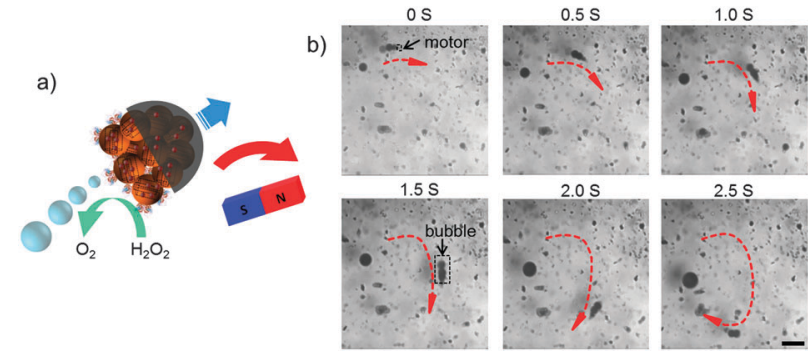

Fig. 4 (a) Schematic illustration and (b) video snaps of bio-catalytic reaction driven motion of a catalase conjugated JMSC motor under magnetic guidance with $3 w t \% \mathrm{H}_{2} \mathrm{O}_{2}$ (scale bar $=200 \mu \mathrm{m}$ ).

In order to prove the successful conjugation of catalase by EDC-NHS chemistry, as well as its catalytic activity after conjugation, the catalase conjugated JMSC was centrifuged and presented as a small pellet at the bottom of a tube. Then, one drop of $\mathrm{H}_{2} \mathrm{O}_{2}$ solution $\left(10 \mu \mathrm{L}, 30 \%\right.$ ) was added. Once the $\mathrm{H}_{2} \mathrm{O}_{2}$ solution comes in contact with the pellet at the bottom, vigorous bubble generation was observed (Video S1 in the ESI $\dagger$ ). After $24 \mathrm{~h}$ storage at room temperature, the same experiment was repeated. It was found that the catalytic capability of catalase remained similar as before (Video S2 in the ESI $\dagger$ ), suggesting that the bio-catalytic activity of catalase conjugated to the JMSC motors was maintained. Besides, conjugated catalase was not only reusable, but also capable of surviving for a relatively long time, at least up to $24 \mathrm{~h}$ at room temperature.

The bio-catalytically driven motion of the JMSC motor was first tested without magnetic guidance. The JMSC@Ni-catalase was suspended in an aqueous solution containing $3 \mathrm{wt} \% \mathrm{H}_{2} \mathrm{O}_{2}$ which was used as the fuel. Bubble generation against the movement direction was clearly observed (Video S3 in the ESI $\dagger$ ), moving from the left to the right (at the top left of the video). As expected, the JMSC motor moved in a straight pathway, heading the side of the Ni coating layer, while the bubbles are generated from the catalase conjugated side, providing the driving force for the movement by the bubble propulsion mechanism.

Then, an external magnetic field was applied by using a permanent magnet, illustrated in Fig. 4a. Under magnetic guidance, the movement direction can be readily changed by varying the orientation of the magnetic field, as the trajectory was shown in Fig. $4 \mathrm{~b}$ by the red curve. The JMSC motor indicated by the black arrow effectively produced bubbles to provide the driving force for the motor. We also observed the motion of the JMSC by bubble propulsion with $\mathrm{H}_{2} \mathrm{O}_{2}$ concentration as low as $1.5 \mathrm{wt} \%$. The lower limit of $\mathrm{H}_{2} \mathrm{O}_{2}$ fuel concentration was found to be about 1 wt\%, under which value bubble generation could barely be observed. Clearly, the JMSC motor was driven by the bubble propulsion mechanism. The bubble generation should be attributed to the rough surface with small cavities at the catalytic side of the JMSC motor. However, even under the same concentration of $\mathrm{H}_{2} \mathrm{O}_{2}$, the bubble generation rate, bubble size and speed of the motors varied a lot (Videos S3 and S4, ESI $\dagger$ ), which can be explained by the non-uniform size and irregular shape of the JMSC motors given by uncontrolled aggregation of MSNPs during the synthesis.
In summary, a bio-catalytically driven JMSC motor with remote magnetic control on motion directionality was successfully produced. Catalase enzyme was anchored to the JMSC motor to provide driving force by decomposition of $\mathrm{H}_{2} \mathrm{O}_{2}$. Taking advantage of the rough surface with small cavities/gaps between aggregated MSNPs, $\mathrm{O}_{2}$ bubbles were effectively generated, resulting in bubble propulsion for the JMSC motor movement. The mesoporous properties of the novel motor were used for cargo loading and therefore the JMSC motor could act as an effective delivery vehicle. The magnetic coating layer (Ni) allows for remote control on the movement direction of the JMSC motor. The possibility of transporting cargo molecules to desired locations by the JMSC motor can be expected. Future research will focus on controlled self-assembly of MSNPs to produce JMSC motors with required size and shape, which will be helpful to realize stable and efficient bubble generation, as well as the controlled speed of the JMSC motor.

This work is financially supported by the European Research Council under the European Union's Seventh Framework Programme (FP7/20072013)/ERC grant agreement [no. 311529], and Alexander von Humboldt Foundation. The authors thank Prof. Peter A. van Aken and Mr Kersten Hahn for assistance with TEM imaging.

\section{Notes and references}

1 S. Sanchez, L. Soler and J. Katuri, Angew. Chem., Int. Ed., 2014, DOI: 10.1002/anie.201406096R1.

2 M. Guix, C. C. Mayorga-Martinez and A. Merkoçi, Chem. Rev., 2014, $114,6285$.

3 A. Brown and W. Poon, Soft Matter, 2014, 10, 4016.

4 T.-C. Lee, M. Alarcón-Correa, C. Miksch, K. Hahn, J. G. Gibbs and P. Fischer, Nano Lett., 2014, 14, 2407.

5 S. Ebbens, D. A. Gregory, G. Dunderdale, J. R. Howse, Y. Ibrahim, T. B. Liverpool and R. Golestanian, EPL, 2014, 106, 58003.

6 W. Duan, R. Liu and A. Sen, J. Am. Chem. Soc., 2013, 135, 1280.

7 W. Duan, M. Ibele, R. Liu and A. Sen, Eur. Phys. J. E: Soft Matter Biol. Phys., 2012, 35, 1.

8 Y. Wang, R. M. Hernandez, D. J. Bartlett, J. Bingham, T. R. Kline, A. Sen and T. E. Mallouk, Langmuir, 2006, 22, 10451-10456.

9 W. Gao, X. Feng, A. Pei, Y. Gu, J. Li and J. Wang, Nanoscale, 2013, 5, $4696-4700$

10 M. Manjare, B. Yang and Y. P. Zhao, Appl. Phys. Lett., 2012, 109, 128305.

11 J. G. Gibbs and Y.-P. Zhao, Appl. Phys. Lett., 2009, 94, 163104.

12 S. Wang and N. Wu, Langmuir, 2014, 30, 3477.

13 S. J. Ebbens and J. R. Howse, Langmuir, 2011, 27, 12293.

14 L. Baraban, M. Tasinkevych, M. N. Popescu, S. Sanchez, S. Dietrich and O. G. Schmidt, Soft Matter, 2012, 8, 48-52.

15 J. R. Howse, R. A. L. Jones, A. J. Ryan, T. Gough, R. Vafabakhsh and R. Golestanian, Phys. Rev. Lett., 2007, 99, 048102.

16 L. Zhang and Y. Zhu, Langmuir, 2012, 28, 13201.

17 N. Suzuki, X. Jiang, L. Radhakrishnan, K. Takai, K. Shimasaki, Y.-T. Huang, N. Miyamoto and Y. Yamauchi, Bull. Chem. Soc. Jpn., 2011, 84, 812.

18 P. Borah, X. Ma, K. T. Nguyen and Y. Zhao, Angew. Chem., Int. Ed., 2012, 51, 7756.

19 Q. Zhang, F. Liu, K. T. Nguyen, X. Ma, X. Wang, B. Xing and Y. Zhao, Adv. Funct. Mater., 2012, 22, 5144.

20 X. Ma, C. Teh, Q. Zhang, P. Borah, C. Choong, V. Korzh and Y. Zhao, Antioxid. Redox Signaling, 2014, 21, 707.

21 K. C. W. Wu and Y. Yamauchi, J. Mater. Chem., 2012, 22, 1251.

22 Y. D. Chiang, H. Y. Lian, S. Y. Leo, S. G. Wang, Y. Yamauchi and K. C. W. Wu, J. Phys. Chem. C, 2011, 115, 13158.

23 Z. Li, J. C. Barnes, A. Bosoy, J. F. Stoddart and J. I. Zink, Chem. Soc. Rev., 2012, 41, 2590.

24 M. Xuan, J. Shao, X. Lin, L. Dai and Q. He, ChemPhysChem, 2014, $15,2255$. 
25 Y. Mei, G. Huang, A. A. Solovev, E. B. Ureña, I. Mönch, F. Ding, T. Reindl, R. K. Y. Fu, P. K. Chu and O. G. Schmidt, Adv. Mater., 2008, 20, 4085.

26 A. A. Solovev, Y. Mei, E. Bermúdez Ureña, G. Huang and O. G. Schmidt, Small, 2009, 5, 1688.

27 L. Soler, V. Magdanz, V. M. Fomin, S. Sanchez and O. G. Schmidt, ACS Nano, 2013, 7, 9611.

28 S. M. Harazim, W. Xi, C. K. Schmidt, S. Sanchez and O. G. Schmidt, J. Mater. Chem., 2012, 22, 2878.

29 V. M. Fomin, M. Hippler, V. Magdanz, L. Soler, S. Sanchez and O. G. Schmidt, IEEE Trans. Rob., 2014, 30, 40.

30 B. Jurado-Sánchez, S. Sattayasamitsathit, W. Gao, L. Santos, Y. Fedorak, V. V. Singh, J. Orozco, M. Galarnyk and J. Wang, Small, 2014, DOI: $10.1002 / \mathrm{smll} .201402215$.
31 S.-H. Wu, C.-Y. Mou and H.-P. Lin, Chem. Soc. Rev., 2013, 42, 3862.

32 Q. Zhang, X. Wang, P.-Z. Li, K. T. Nguyen, X.-J. Wang, Z. Luo, H. Zhang, N. S. Tan and Y. Zhao, Adv. Funct. Mater., 2014, 24, 2450.

33 S. Sanchez, A. A. Solovev, Y. Mei and O. G. Schmidt, J. Am. Chem. Soc., 2010, 132, 13144.

34 F. Q. Tang, L. L. Li and D. Chen, Adv. Mater., 2012, 24, 1504.

35 Q. He and J. Shi, J. Mater. Chem., 2011, 21, 5845.

36 X. Ma, O. S. Ong and Y. Zhao, Biomater. Sci., 2013, 1, 912.

37 B. M. Cash, L. Wang and B. C. Benicewicz, J. Polym. Sci., Part A: Polym. Chem., 2012, 50, 2533.

38 Y. An, M. Chen, Q. Xue and W. Liu, J. Colloid Interface Sci., 2007, 311, 507. 